\title{
Monad Theory of Structural Synthesis of Mechanisms
}

\author{
Sergey Kuznetsov ${ }^{1, *}$ \\ ${ }^{1}$ Institute of Fundamental Engineering Education, Platov South-Russian State Polytechnic University \\ (NPI), Novocherkassk, 346428, Russian Federation
}

\begin{abstract}
Structural synthesis creates a structural scheme according to the given structural characteristics - the degree of abnormality of the structure $S$ and the degree of its irrationality $s$. Structural scheme is created at the first stage of synthesis, containing only rotational kinematic pairs and corresponds to a given degree of abnormality, which is ensured by the ratio of structural units - plus monads, minus monads and null monads. At the second stage, the independence of structural characteristics makes it possible to bring the obtained scheme to a given degree of irrationality by downgrading the class of kinematic pairs. The proposed algorithm makes it possible to synthesize structural schemes of both normal structure and adaptive (with redundant motions), as well as indifferent (with redundant links). Monadic approach to structure research allows to formalize the structural features of the circuit and to create a knowledge base for automated design of structural circuits with given structural properties.
\end{abstract}

\section{Monads as structural units}

The existing structural theories of synthesis are exclusively limited to layering of normal structure groups $[1,2,3]$, although the same L.V. Assur suggested the possibility of studying cases of "structural abnormalities" [4]. The practice of structural analysis shows that not all mechanisms have a regular structure; moreover, mechanisms of adaptive structure are rather widely spread (differentials) and examples and properties of indifferent structures are shown in $[5,6]$.

To solve the problem of synthesis of any structures, including abnormal (non-Assur type) structures, it is necessary to distinguish elementary structural units, that, like molecules from atoms, comprise structural groups of different abnormalities. For this purpose the abnormality degree formula [6]

$$
S=3 n-2\left(p_{5}+p_{4}+p_{3}\right)-p_{2}-p_{1}-p_{0},
$$

where $n$ is the number of moving elements;

$p_{5}$ - the number of kinematic pairs of class 5 (turning and sliding);

$p_{4}$ - the number of kinematic pairs of class 4 (cylindrical);

$p_{3}$ - the number of kinematic pairs of class 3 (spherical);

$p_{2}$ - the number of kinematic pairs of class 2 (linear);

\footnotetext{
* Corresponding author: sergey-kuznecov-57@mail.ru
} 
$p_{1}$ - the number of kinematic pairs of class 1 (point);

$p_{0}$ - the number of input links superimposed on the input elements and marked by an arrow; this should be simplified, thus leaving in the formula only kinematic pairs of the fifth class, and converting it to the following form

$$
p=\left(3 n-S-p_{0}\right) / 2 \text {. }
$$

According to this formula, the simplest single element chain of normal structure $S=0$ will be a null monad, or an input element, more precisely, the element with one turning pair, on which an input link is superimposed (fig. 1, $a$ )

$$
p=(3 \cdot 1-0-1) / 2=1 \text {. }
$$

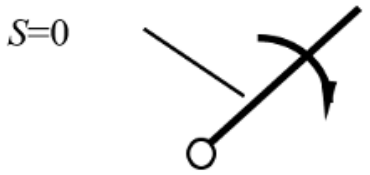

a)

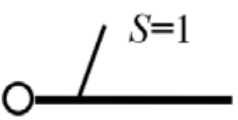

b)

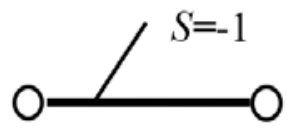

c)

Fig. 1. Monads with different abnormality grades: a) monad; b) plus monad; c) minus monad

The simplest chain of adaptive structure $S=1$ will be guided at $p_{0}=0-$ plus monad (see fig. $1, b)$ :

$$
p=(3 \cdot 1-1-0) / 2=1 \text {. }
$$
$1, c)$ :

The simplest chain of indifferent structure $S=-1$ at $p_{0}=0$ will be minus monad (see fig.

$$
p=(3 \cdot 1+1-0) / 2=2 .
$$

All other chains of normal structure (called Assur groups), as well as abnormal structure chains with bilateral bonds (to inferior pairs) are combinations of these three monads. For example, a dyad is a combination of a plus monad and a minus monad (see fig. 2, a), a three-rod group is created by three plus monads and three minus monads (see fig. 2, b), and so on.
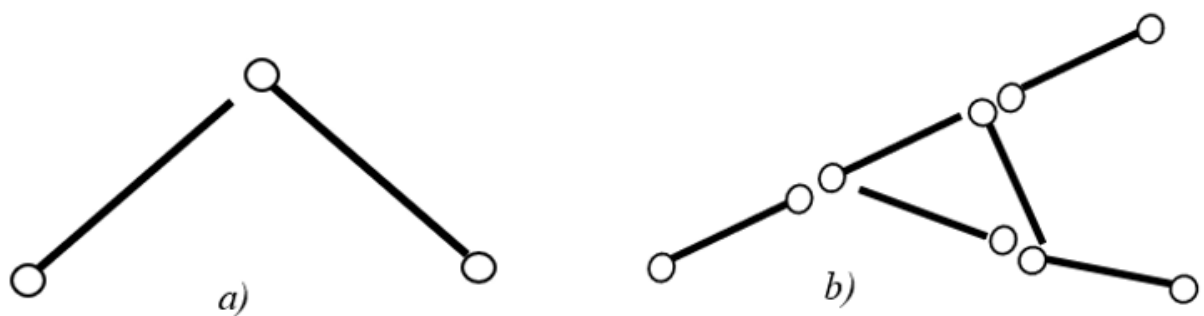

Fig. 2. Groups of regular structure combined of monads: a) dyad; b) three-rod group

Obviously, the regular structure groups are formed by equal numbers of plus and minus monads, while abnormal structure chains are formed by combining unequal numbers of plus and minus monads. 


\section{Formalization of structural features}

Synthesis in the general case is an inverse process of analysis: the output parameters of synthesis are the input parameters of analysis, and vice versa. Obviously, with this formulation of the question, the input parameters at synthesis should be the structural characteristics $S$ and $s$, and the output parameters should include the quantity of elements and pairs of each class; in this case, the output parameters of synthesis are more than the structural formulas. To successfully solve the synthesis problem, it is necessary to increase the number of structural dependences due to formalization of structural features that are not included in the number of basic structural parameters, such as: the number of rigid and movable circuits, that can be internal and external (adjacent to the rack); multiplicity of kinematic pairs; number of stationary pairs linking the elements with the rack, and so on.

Since monads are the prime structural units, rigid contours are also formed by monads: a triangle is formed by three monads, a quadrangle by five monads, a pentagon by seven monads (see fig. 3), etc.:

$$
m_{i}=2 a_{i}-3,
$$

where $m_{i}$ stands for the quantity of monads forming each rigid circuit, found from the condition of its kinematic determinability.
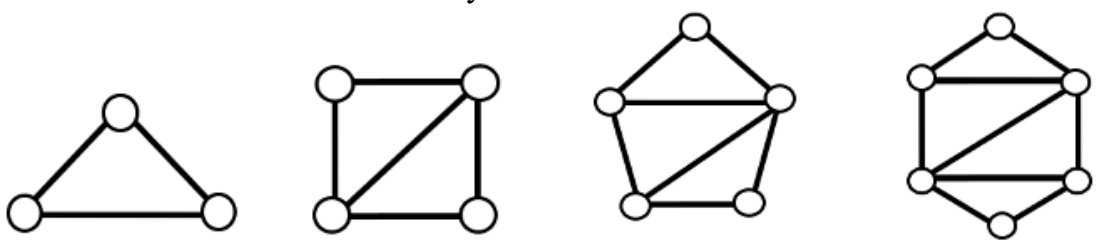

Fig. 3. Rigid closed circuits

If the number of independent circuits can be determined by H.I. Gokhman's formula: $\mathrm{c}=p-n$, then on the monadic level the number of all contours, including triangular, making up the rigid circuits, is determined by a similar formula:

$$
c_{m}=p_{m}-m,
$$

where $m$ is the quantity of monads (lines), that form the structure;

$p_{m}$ is the quantity of kinematic pairs on monad level or total multiplicity of kinematic pairs.

The projection of the structural scheme on the plane consists of plus and minus monads connected in a certain way. Simply stated, these are circles, which may simultaneously include several kinematic pairs, and lines connecting them. The number of kinematic pairs in one circle, or multiplicity of a kinematic pair, is one unit less than the number of lines that converge in it. Then the number of triangular circuits in the structure (triangles) is equal to the difference between the number of circuits on the monadic level (2) and the number of moving circuits according to the Gokhman's formula:

$$
\Delta=c_{m}-c=p_{m}-m-k .
$$
study:

The formula (1) is represented in the following way on the monad level of a structure

$$
S=3 m-2 p_{m}-p_{0}
$$

from which

$$
p_{m}=\left(3 m-p_{0}-S\right) / 2 \text {. }
$$


After the substitution of $p_{m}$ into the formula (3) we obtain

$$
m=S+p_{0}+2(c+\Delta) \text {. }
$$

This formula relates the number of monads $m$ to the number of input elements $p_{0}$, the degree of structure abnormality $S$, the number of moving circuits $c$, and the number of triangles forming rigid circuits $\Delta$. We obtain from the formulas (1) and (5) the number of moving elements $n$, expressed in terms of the number of moving circuits $c$ :

$$
n=S+p_{0}+2 c .
$$

The number of kinematic pairs is determined from the formula (1):

$$
p=\left(3 n-p_{0}-S\right) / 2 \text {. }
$$

The total number of redundant multiples of kinematic pairs, showing how many lines converge in circles, except the two put for a single pair, we define as the difference between the number of pairs at the monadic level (2) and the number of pairs at the elements level (1):

$$
p_{\Sigma}=p_{m}-p
$$

Each movable circuit adjacent to the rack is connected to it by two stationary pairs; each new circuit gives one more stationary pair. Thus, the number of fixed pairs linking the elements of the mechanism with the rack is one more than the number of such circuits minus the inner movable circuits, which are not adjacent to the rack:

$$
p_{s}=c+1-c_{i}
$$

where $c_{i}$ stands for the inner movable circuits, which are not adjacent to the rack.

The number of triangles in a branched (complex) circuit is one less than the number of moving circuits $c$, except for internal $c_{i}$ :

$$
\Delta=c-1+c_{i} .
$$

We obtain the following after the substitution in (5):

$$
m=S+p_{0}+2\left(2 c-1+c_{i}\right)=2(2 p-\Delta-2)-3 n .
$$

The quantity of plus monads is determined by the formula

$$
+m=\left(m+S-p_{0}\right) / 2 .
$$

The quantity of minus monads is determined by the formula

$$
-m=\left(m-S-p_{0}\right) / 2 \text {. }
$$

All formulas that make up the knowledge base for structural synthesis are derived by comparing the corresponding parameters at the monadic level and at the level of whole elements. With the help of these formulas it is possible to obtain the basic parameters of the synthesis of structural circuits and additional - structural features.

\section{Stages and algorithms of structural synthesis}

The use of the knowledge base, based on the monadic structure theory, allows synthesizing the structural scheme in two stages.

At the first stage, using the above obtained formulas, the output parameters of the structural scheme synthesis are calculated according to the given degree of abnormality and certain structural features. If the structural features are not specified, that is, are not 
included in the number of input synthesis parameters, their value is taken as equal to the minimum possible. For example, the minimum value of number of moving circuits $c=1$; in this case there cannot be any triangles, there cannot be multiples of kinematic pairs, and so on. If the single-loop circuit does not pass the kinematic or dynamic conditions, as it will be found out in further calculations, the designer or CAD system should return to the first stage in order to vary the structural features until a satisfactory result is obtained. At the same stage, part of the bilateral kinematic pairs is replaced, if necessary, by unilateral kinematic pairs in the proportion of two to one with reduction of the number of moving elements in inverse proportion to the number of the introduced higher pairs.

At the second stage, the obtained structural scheme consisting of circles connected by lines is adjusted to a given degree of irrationality. At this stage, the contour redundant connections are eliminated according to the certain rules:

- if all redundant connections are eliminated by structural means, it is necessary to check the correctness of the replacements of kinematic pairs by contour-by-contour determination of the degree of irrationality;

- if not all redundant connections are eliminated, the output circuit, being most loaded circuit, gets rid of them to the greatest extent;

- in the case of a non-standard assignment of the degree of irrationality, for example, positive (for self-setting), it is necessary to control that the excessive mobilities should be:

a) local, but not grouped, and

b) not related to the output element, but were located within the output circuit.

Consider an approximate algorithm for calculation of the parameters of the structural diagram with the following combination of input synthesis parameters:
abnormality grade of the structure
input elements (links) quantity
moving circuits quantity
$S=0$
$p_{0}=1$
$c=3$,

which means a mechanism of regular structure with one input element and three moving circuits. In this case it does not matter how many and which Assur groups will be in this structure.

1). According to the formula (6) we calculate the number of moving elements:

$$
n=S+p_{0}+2 c=0+1+2 \cdot 3=7 .
$$

2). According to the formula (7) we determine the number of kinematic pairs $p$ :

$$
p=\left(3 n-p_{0}-S\right) / 2=(3 \cdot 7-1-0) / 2=10 .
$$

The main synthesis parameters are found, but they are not enough to build a structural scheme. It is necessary to know the remaining structural features.

3 ). We determine the number of monads that make up the structural scheme (11)

$$
m=S+p_{0}+2\left(2 c-1+c_{i}\right)=0+1+2(2 \cdot 3-1+0)=11 .
$$

4). The number of pairs on the monadic level (4)

$$
p_{m}=\left(3 m-p_{0}-S\right) / 2=(3 \cdot 11-1-0) / 2=16 .
$$

$5)$. The number of stationary pairs in the absence of internal moving circuits is determined by the formula (9)

$$
p_{s}=c+1-c_{i}=3+1-0=4 .
$$

6). The total number of excess multiples of kinematic pairs is determined by the formula (8)

$$
p_{\Sigma}=p_{m}-p=16-10=6 \text {. }
$$


7). The number of triangles is determined according to the formula (10) (fig.4)

$$
\Delta=c-1+c_{i}=3-1+0=2 .
$$

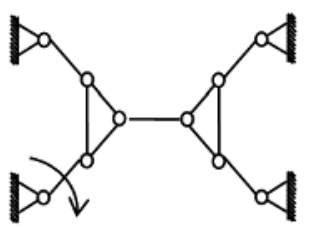

a)

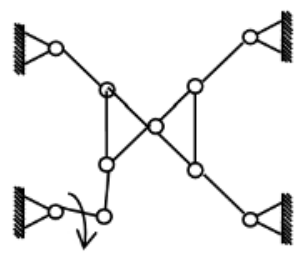

b)

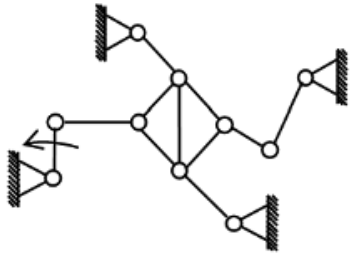

c)

Fig. 4. Variants of structural diagrams with two triangles

\section{References}

1. $\quad$ P L Chebyshev On Parallelograms, Complete Works of P.L. Chebyshev, Vol.4 (M.L.: Publ. of the Academy of Sciences of the USSR, 1948)

2. I I Artobolevsky Theory of Mechanisms and Machines: Textbook for Technical Colleges (M.: Nauka. G.red. fiz.mat. lit.,1988)

3. K V Frolov, S A Popov, A K Musatov, G A Timofeev Theory of Mechanisms and Mechanics of Machines: Textbook for Technical Colleges, ed Frolov K V (M.: Vyssh.shk., 2001)

4. L V Assur Study of Flat Bar Mechanisms with Inferior Pairs in Terms of their Structure and Classification S A (Moscow: Publishing House of the Academy of Sciences of the USSR, 1952)

5. S A Kuznetsov IOP Conference Series: Materials Sci., Engl. 709, Issue 2 (2019)

6. S A Kuznetsov IOP Conference Series: Materials Sci., Engl. 971042008 (2020) 